\title{
Gobierno local, planeación y gestión de los servicios públicos en ciudades medias de México. El caso de la zona metropolitana de San Luis Potosí*
}

\author{
Adrián Moreno Mata \\ Universidad Autónoma de San Luis Potosí
}

\section{Resumen}

En esta investigación se pretende explorar de manera conceptual y empírica la experiencia concreta de la gestión de los servicios públicos en las ciudades medias deMéxico, y las complejidades administrativas, técnicas e institucionales que surgen a partir de su prestación, especialmente en un marco en el que cada vez más el ciudadano común de estas ciudades no se cuestiona acerca de las grandes orientaciones de la política urbana, sino sobre las deficiencias o aciertos de la función gubernamental en la cobertura y calidad de estos servicios, y su efecto en las condiciones de vida y el nivel de satisfacción que obtiene.

continúa

\footnotetext{
- Este artículo es una versión - corregida y aumentada- del trabajo denominado "Gobierno metropolitano y administración de los servicios públicos en San Luis Potosí: 19901997”, presentado en el Segundo Congreso de Investigación Urbana y Regional: Balance y Perspectivas, organizado por la Red Nacional de Investigación Urbana, en la semana del 29 de septiembre al 3 de octubre de 1997, en la ciudad de Tlaxcala, Tlax., México.
} 
Para ilustrar lo anterior, se elabora una somera evaluación del desempeño de las administraciones municipales en el caso de la zona metropolitana de San Luis Potosí, en un periodo que abarca de 1990 a la fecha, que corresponde a una etapa caracterizada por la alternacia en el gobierno de esta ciudad, entre el partido oficial -el PRI-, el PAN y otros partidos o coaliciones. Se ofrece así un panorama general sobre los principales obstáculos y limitaciones que han enfrentado dichas administraciones para una adecuada planeación, administración y prestación de los servicios públicos.

Se concluye que esta alternancia política no ha significado, en el caso de estudio, una mejor gestión de los servicios públicos, ni cambios sustantivos en materia de autonomía, descentralización, y eficiencia administrativa o técnica. Por el contrario, la información disponible revela que, independientemente del origen partidista del gobierno local, las características e inercias de su estructura interna y el predominio de un modelo de gestión de corte tecno-burocrático, han impedido enfrentar con relativo éxito las deficiencias que presentan la cobertura y dotación de los servicios de agua potable y saneamiento.

Finalmente, se plantean algunas reflexiones sobre alternativas innovadoras que podrían mejorar la capacidad de la administración local para enfrentar la problemática descrita, destacando entre ellas el diseño de una política pública urbana basada en un modelo de gestión de los servicios públicos de carácter participativo, entendido como un proceso dinámico de confrontación de proyectos, articulación de esfuerzos y solución de conflictos entre intereses $y / o$ actores sociales.

\section{Introducción}

En distintos ámbitos es cada vez más notorio el interés por analizar el desempeño de los gobiernos municipales en la solución de necesidades y demandas sociales, especialmente en aquellos casos en los que sus formas de actuación se ven seriamente afectadas, cuando a las tradicionales tareas que impone la administración municipal se suman las derivadas del crecimiento urbano, y aún más, cuando éste deriva hacia una expansión territorial de carácter metropolitano.
Adicionalmente, la situación actual por la que atraviesa un gran número de municipios en México revela una clara contradicción, ya que por un lado, sabemos que gran parte de la obligación de atender las necesidades de servicios públicos básicos -como agua potable, drenaje y basura- recae precisamente en los municipios, mientras que por el otro, éstos constituyen el nivel de gobierno con mayores carencias de recursos. De hecho, esto que parece tan simple, ilustra de manera muy cruda la realidad municipal, pues independientemente del grado de modernización administrativa, de innovaciones en la gestión y organización municipales, o de nuevas formas de relación entre los gobiernos locales y las organizaciones sociales, el factor que determina los alcances o limitaciones del ejercicio gubernamental en este ámbito es la enorme dependencia de la estructura financiera municipal; situación que se agrava en el difícil panorama que enmarcan la crisis fiscal del estado y la crisis del Estado Benefactor, cuyas secuelas en México comienzan a tomar visos realmente alarmantes desde la década de los ochenta. ${ }^{1}$

No obstante, existe un cierto consenso en torno a la idea de que la descentralización del Estado, la redistribución de funciones y su entrega a los niveles periféricos de gobierno, podrían propiciar una mayor eficacia y, eventualmente, un desempeño más eficiente en la prestación de los servicios públicos. Esto sumado a una mayor participación ciudadana en la gestión municipal, aspecto que nos remite al análisis de las relaciones entre el municipio, como instancia de gobierno, los problemas de la ciudad y la intervención de los distintos actores que participan e inciden en la vida local. ${ }^{2}$

En este contexto, destaca el triple papel que juegan los servicios públicos en la coyuntura urbana: como punto de contacto fundamental entre gobernantes y gobernados; como elemento clave

' Al respecto, pueden consultarse los trabajos publicados por E. Gutiérrez Garza (ed.), Los saldos de la crisis México, Fondo de Cultura Económica, 1990; y A. Moreno Mata “Los límites del Estado benefactor: Ciencis Sociales y Humanidades de la Universidad Autóno enero-agosto, 1991, pp. 141-145.

${ }^{2}$ Resulta aquí importante precisar la definición de dos conceptos: gestión y administración del desarrrollo. Conceptos que en su definición clásica habían estado vinculados, del desarrollo. Conceptos que en su definicion clásica hablan estado vinculados, indanentmente, con la práctica gubernato de ha planeacion, considerandose indistintamente a uno como funcion del otro, pero que a la luz de los nuevos enfoques aparecen claramente diferenciados. La gestion, como una acción que lleva a cabo tanto la sociedad civil en su conjunto, como el gobierno de las localidades, mientras que la administración responde sobre todo a las necesidades del sector público de dirigir -esto es, recabar, manejar, destinar, asignar los recursos y/o fondos públicos- en concordancia con las políticas, los programas y objetivos de gobierno. 
para el desarrollo municipal y metropolitano; y como herramienta básica para la planeación urbana de los gobiernos locales. En este sentido, debe reconocerse que el estudio de la gestión local -entendida como la organización y el manejo de una serie de recursos institucionales, financieros, humanos y técnicos, provenientes de los tres niveles de gobierno, para atender necesidades de la población-, comprende diversos elementos de naturaleza metodológica, algunos de los cuales se mencionan en seguida:

En primer lugar deben considerarse tres dimensiones: $\imath$ ) los grados de autonomía política y administrativa del municipio, es decir, la capacidad que éste tiene para tomar decisiones indispensables en la atención de necesidades sociales, sin la intervención de otros agentes privados o públicos extramunicipales; ii) la eficacia y eficiencia de la gestión, esto es, de qué manera se emplean los recursos para atender dichas necesidades y cuál es la capacidad de generar programas que tengan un impacto positivo en las condiciones de vida de la población demandante; y iii) las relaciones entre los actores involucrados en la gestión, las cuales inciden definitivamente en el grado de legitimidad del gobierno municipal, en el funcionamiento de las instancias de representación, y en la existencia de canales de participación política y ciudadana que fomenten los vínculos entre gobierno local y ciudadanía. Una dimensión más puede apoyar este abordaje, y se refiere a la gestión municipal como forma de control políticoinstitucional. Al respecto, debe destacarse la poca experiencia de gestión de los gobiernos locales por parte de los partidos políticos. Aunque pueden registrarse algunos procesos aislados de inducción de innovaciones en materia de gobierno, administración y gestión municipal en México (Cabrero, 1995), por lo general el tipo de gestión que conocemos en México es una mezcla de autoritarismo unipersonal -centralizado en la figura del alcalde-, eficiencia empresarial, y relaciones clientelistas con los distintos sectores sociales y políticos que actúan en el nivel local.

Esto nos lleva, en el marco de un creciente pluralismo y participación democráticos y de un fenómeno cada vez más presente en los municipios como es la alternancia política, a impugnar la capacidad de los nacientes partidos de oposición para responder a los retos del ejercicio del poder en los gobiernos locales urbanos; y a replantear las implicaciones de estos procesos para favorecer una efectiva descentralización política y administrativa.

De igual forma, es necesario mencionar, aunque sea de paso, la diversidad de factores que han limitado y aún limitan la actuación administrativa y planificadora de los municipios mexicanos, independientemente de los procesos del nuevo federalismo y la tendencia descentralizadora del Estado, y que en materia deprestación delosservicios públicos municipales seilustran ampliamente: ausencia de objetivos y plazos de realización, confusión de objetivos con propósitos o valores deseados, poca orientación de los diagnósticos a establecer el contexto y las causas de los problemas, y un señalamiento casi nulo referente a la necesidad de control y evaluación. Aspectos que hacen muy complejo - por no decir imposible- que las administraciones municipales asuman por lo menos con relativo éxito las nuevas facultades y funciones que generosamente comienza a cederles el gobierno central. De ahú la urgencia de incorporar análisis que permitan emitir ciertos indicadores o parámetros de evaluación acerca del posible éxito o del eventual fracaso de estas tendencias.

En el presente artículo se analiza la experiencia concreta de la gestión de los servicios públicos en las ciudades medias de México, y sobre las complejidades administrativas, técnicas e institucionales que surgen a partir de su prestación, especialmente en un marco en el que cada vez más el ciudadano común de estas ciudades no se pregunta respecto a las grandes orientaciones de la política urbana, sino sobre las deficiencias o aciertos de la función gubernamental en la cobertura y calidad de estos servicios, y su efecto en las condiciones de vida y el grado de satisfacción que obtiene.

Con este trabajo se pretende evaluar la capacidad del gobierno de la zona metropolitana (ZM) de San Luis Potosí en materia de planeación, administración y gestión de los servicios básicos. El periodo de estudio abarca de 1990 a 1997, y corresponde a una etapa caracterizada por la alternacia en el gobierno de esta ciudad entre el PRI (Guillermo Medina de los Santos [1988-1990] y Luis García Julián [1995-1997]), el PAN (Guillermo Pizzuto Zamanillo [1990-1992] y Mario Leal Campos [1992-1994]), o coaliciones como la del Consejo Ciudadano Interino (Rafael del Blanco [1995]).

En el primer apartado se analizan diversos indicadores sobre la calidad y cobertura de los servicios públicos urbanos en el ámbito metropolitano. También se presentan datos relativos al desarrollo desigual en su dotación, en demérito de las condiciones de vida de población segregada social y espacialmente; para tal efecto, se incluye información de las características de servicios públicos básicos en más de 150 vecindades, ubicadas en torno al centro histórico de la ciudad.

Posteriormente se elabora una somera evaluación del desempeño delas administraciones municipales estudiadas, ofreciendo un panorama general sobre los principales obstáculos y limitaciones que han enfrentado para una adecuada planeación y gestión de los 
servicios públicos. De manera tentativa, se plantea que esta alternancia política no ha significado una mejor prestación de los servicios de agua potable y saneamiento, ni cambios sustantivos en los gobiernos locales en materia de autonomía, descentralización, y eficiencia administrativa o técnica. Por el contrario, la información disponible revela que, independientemente de su origen partidista, las características e inercias de su estructura interna y el predominio de un modelo de gestión de corte tecno-burocrático, les han impedido atender con relativo éxito los déficit observados.

El artículo concluye con algunas reflexiones sobre opciones innovadoras que podrían mejorar la capacidad de las administraciones locales para enfrentar la problemática descrita, destacando entre ellas el diseño de una política pública urbana basada en un modelo de planeación y gestión delos servicios públicos de carácter participativo, entendido como un proceso dinámico de confrontación de proyectos, articulación de esfuerzos y solución de conflictos entre intereses y/o actores sociales

\section{Los servicios de agua potable y drenaje en la ZM de San Luis Potosí}

\section{Antecedentes}

La evolución de la ZM de San Luis Potosí constituye la expresión concreta del desarrollo de diversas actividades predominantes: en una primera etapa, que va desde la época de la Colonia hasta el periodo del porfiriato, la minería constituyó el eje fundamental del dinamismo económico, papel que posteriormente desempeñaría la actividad comercial hasta bien entrado el siglo XX.

El periodo posrevolucionario marca el inicio de una segunda etapa, en la que se sentaron las bases de importantes transformaciones que irían conjuntando las condiciones que permitieron su despunte económico y la concentración de población. A partir de 1940 y durante las cuatro décadas siguientes, se produjo una creciente expansión demográfica y de la mancha urbana, ligadas al impulso de las actividades industriales, y a finales de ese periodo tuvo lugar el fenómeno de la metropolización, al conurbarse el municipio central de San Luis Potosí con la localidad aledaña de Soledad de Graciano Sánchez (véase el mapa 1).

\section{Mapa 1}

Municipio de San Luis Potosí.

Localización de la ZM, 1996

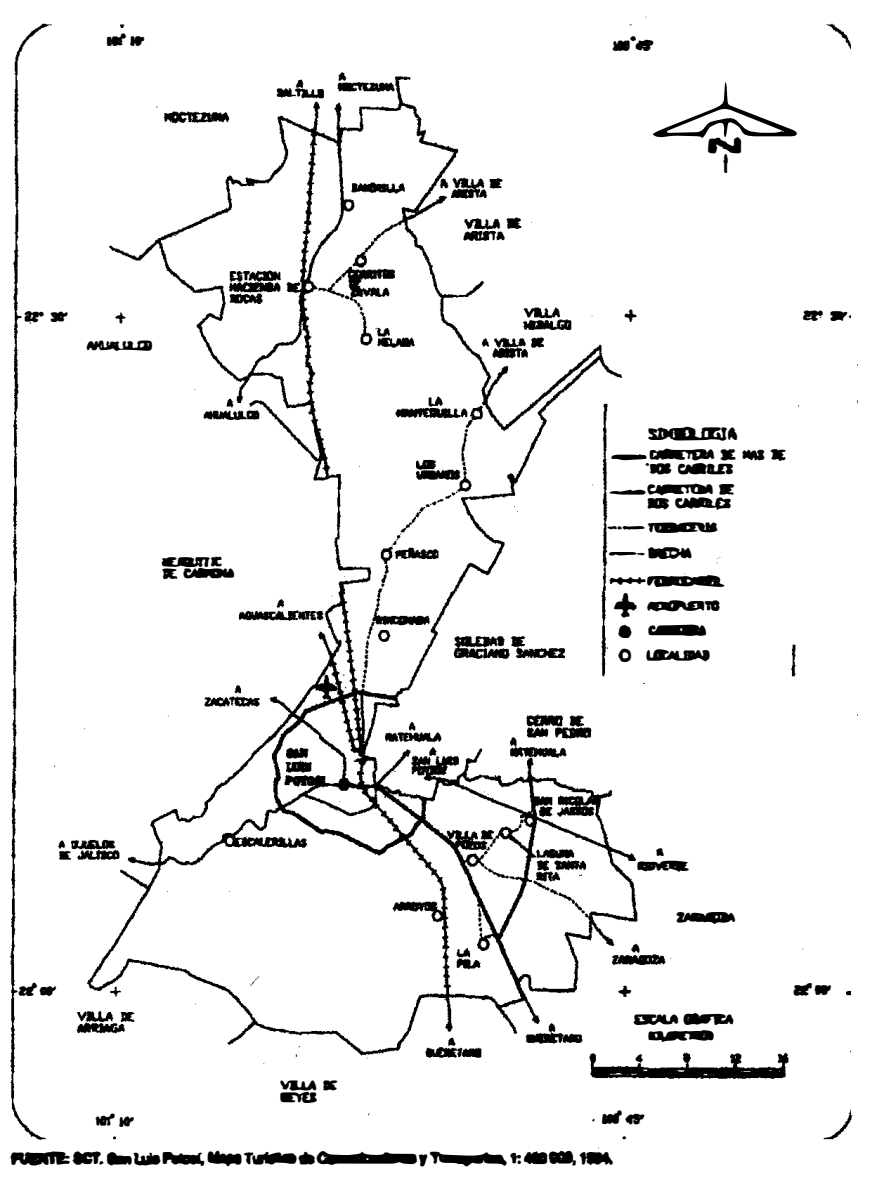


En la etapa actual, que inicia en los años ochenta, la expansión urbana se aceleró de manera notoria, absorbiendo otras localidades; paralelamente, la estructura económica urbana se diversificó, de tal forma que otras actividades, como el comercio y los servicios, comenzaron a competir con la industria en términos de su impacto y relevancia local, dando lugar al fenómeno conocido como la terciarización. Dicha competencia abarcó también la demanda de suelo y servicios básicos, fundamentales para su desenvolvimiento.

En este contexto, el crecimiento de la población residente en la ZM de San Luis Potosí, y el consecuente incremento en la demanda de servicios públicos tales como agua potable, drenaje, recolección de basura, electricidad y alumbrado público, constituyen el principal detonador de una política urbana reactiva implantada por los gobiernos locales, cuya capacidad de planeación, gestión y prestación de esos servicios se ve rebasada constantemente.

Como se observa en el cuadro 1 , según datos censales, a lo largo de ese periodo la población aumentó de 206,261 habitantes en 1960 , a aproximadamente 800,000 en 1995 , con un incremento promedio de 69,000 habitantes cada tres años, que a su vez representaron cerca de 15,000 tomas o descargas domiciliarias.

Cuadro 1

ZM de San Luis Potosí.

Población, superficie y densidad demográfica, 1960-1995

\begin{tabular}{||cccc|}
\hline Año & Población* & $\begin{array}{c}\text { Superficie } \\
\text { (hectáreas) }\end{array}$ & $\begin{array}{c}\text { Densidad bruta } \\
\text { (habitantes/hectárea) }\end{array}$ \\
\hline 1960 & 206,261 & 1,760 & 117.1 \\
1970 & 267,951 & 2,560 & 104.6 \\
1980 & 471,047 & 5,876 & 80.1 \\
1990 & 658,712 & 11,332 & 58.1 \\
1995 & 781,964 & 15,215 & 51.3 \\
\hline
\end{tabular}

Fuente: (*) VII Censo General de Población, DGE, SIC, 1963; IX Censo General de Población, SIC, 1971; x y X Censos Generales de Población y Vivienda, INEGI, 1984 y 1992; Conteo 1995, INEGI, 1996. (**) Dirección General de Catastro del Ayuntamiento de San Luis Potosí, 1962, CETENAL, 1970; Dirección General de Catastro del Gobierno del Estado de San Luis Potosi, 1982; SEDUE y Gobierno del Estado de San Luis Potosí, 1988; Anuario Estadistico del Municipio de San Luis Potosí, INEGI, 1997.
Es importante subrayar que, en esa misma etapa, la extensión física del área metropolitana alcanzó también una magnitud inusitada, elevándose de 1,760 hectáreas en 1960, a más de 15,000 en 1995 , razón de 1,615 hectáreas por trienio. Independientemente del descenso constante en la densidad bruta (habitantes por hectárea) que esto significó, lo relevante de estas cifras es que, considerando la superficie original de San Luis Potosí al principio de la década de los sesenta, ésta se duplicó prácticamente en cada trienio. ${ }^{3}$

\section{Cobertura y calidad de los servicios de agua potable y drenaje}

Es obvio que la influencia de este desmesurado crecimiento afectó de manera creciente la capacidad administrativa, técnica y financiera de los gobiernos municipales encargados de atender la demanda de servicios en el área metropolitana, situación que se ilustra en los datos del cuadro 2. Aunque el periodo 1980-1990 revela un incremento notable en la cantidad y el porcentaje de población atendida por la red de agua potable, que ascendió de 88.5 a $96.1 \%$, entre 1990 y 1995 dicha proporción prácticamente se estancó.

Una tendencia similar se observa al comparar el porcentaje de la población que disponía del servicio dentro de su vivienda, el cual se mantuvo entre 89.5 y $90 \%$ en ese mismo lapso. De cualquier forma, lo relevante del caso es que en 1995 la dotación de este servicio dejaba fuera a casi 30 mil habitantes de la $\mathrm{ZM}$, mientras que $10 \%$ (cerca de 75 mil habitantes) todavía no contaba con servicio dentro de su morada

Sin embargo, esta información poco nos dice del abastecimiento respecto a la calidad del líquido o a la regularidad de servicio. Desde hace varios años, prácticamente todos los sectores de la ciudad se ven afectados periódicamente por racionamientos de este servicio, decretados por el organismo operador descentralizado. De hecho, solamente $87.5 \%$ de la superficie quecubre el sistema hidráulico

${ }^{3}$ Conviene señalar que el crecimiento constante de la mancha urbana en esta zona metropolitana se encuentra asociado de manera importante con una exacerbada especulación en el mercado del suelo. Sin embargo, también responde a una marcada deficiencia de carácte récnico-administrativa que padecen, casi de manera permanente, los gobiernos municipales de San Luis Potosí y Soledad de Graciano Sánchez. En consecuencia, un gran número de fraccionamientos se han construido de manera irregular, sin cumplir con los requisitos básicos en materia de servicios de agua potable, alcantarillado, alumbrado, y servicios de limpia; en e caso del municipio de la capital, durante la administración de Mario Leal Campos (1992-1994) se autorizó la construcción de 102 fraccionamientos, de los cuales aún 82 no cumplen con las disposiciones normativas. También en las administraciones de Rafael-del Blanco y de Luis García.Julián se ha presentado este problema, aunque en menor número de casos. 

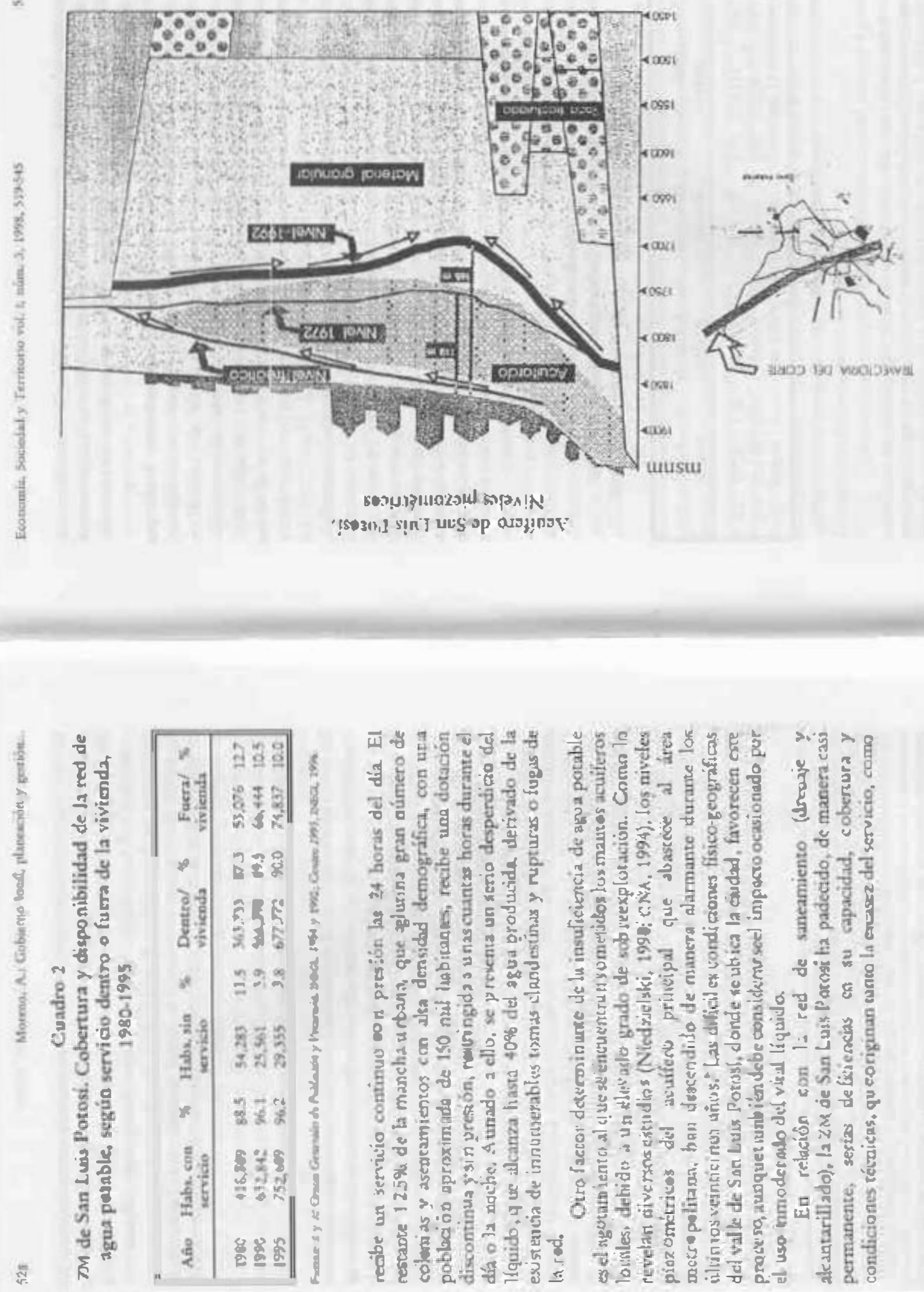

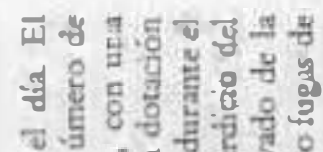

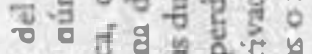

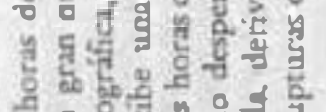

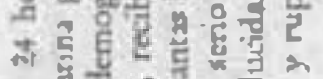

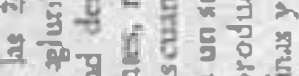

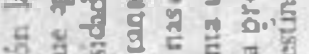
i⿱

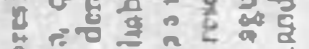
व 8 ज 50 क उ

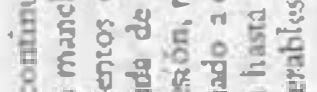
8,5 . 을 क व ํำ รา भ้ ग है

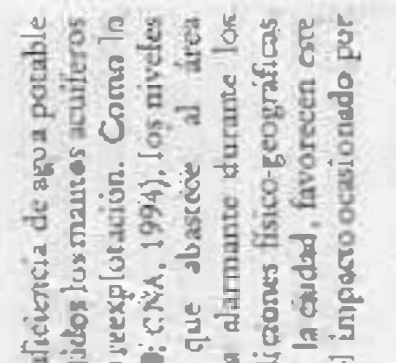

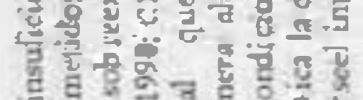

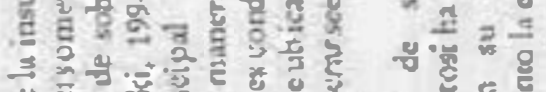

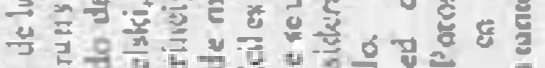

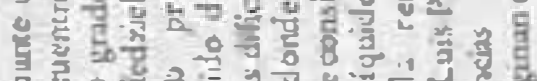
至

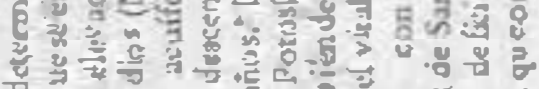

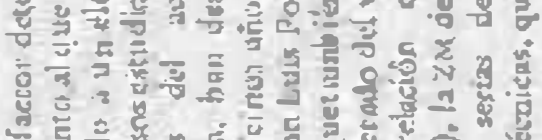

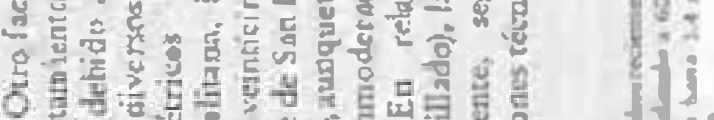

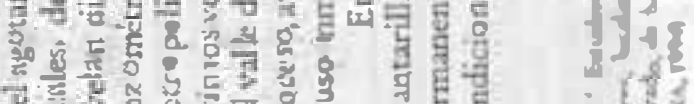

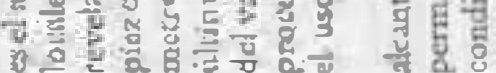


un acelerado proceso de contaminación por descargas domiciliarias al aire libre y desechos industriales. Esto provoca también importantes daños a la salud de la población y daños irreparables en el medio ambiente.

Al respecto, cabe señalar que, prácticamente desde sus orígenes, la expansión urbana de esta ciudad ha enfrentado difíciles condiciones para la prolongación y el tendido de la red de saneamiento. En la actualidad, la obsolescencia de la infraestructura existente, cuyo primer colector data de mediados del siglo $\mathrm{XIX},{ }^{5}$ ocasiona de manera cíclica importantes inundaciones en diversos sectores de la mancha urbana, producto de la derrama pluvial en época de lluvias, que sobrecarga la capacidad de la red. Hasta 1997, las inversiones públicas, federales, estatales y municipales, habían evadido o soslayado la modernización del sistema; casi al final de las presentes administraciones estatal y municipal, se han iniciado las obras del proyecto denominado "Colector Vial Reforma", que pretende subsanar esta carencia, cabe decir, sin la guía de un plan maestro. Independientemente del supuesto beneficio que estas obras puedan generar a partir de su conclusión, las evidencias estadísticas que aparecen en el cuadro 3 expresan la gravedad del efecto acumulado de postergar durante tantos años la atención a este problema.

Cuadro 3

ZM de San Luis Potosí. Cobertura y disponibilidad de la red de drenaje, según tipo de desagüe, 1980-1995

\begin{tabular}{||cccccccccc|}
\hline Año & $\begin{array}{c}\text { Habs. con } \\
\text { servicio }\end{array}$ & $\begin{array}{c}\text { Habs. sin } \\
\text { servicio }\end{array}$ & $\begin{array}{c}\text { A drenaje } \\
\text { público }\end{array}$ & $\begin{array}{c}\text { A fosa } \\
\text { o suelo }\end{array}$ & $\%$ \\
\hline 1980 & 355,923 & 75.6 & 115,124 & 24.4 & 349,150 & 98.1 & 6,773 & 1.9 \\
1990 & 590,205 & 89.6 & 68,506 & 10.4 & 571,605 & 96.8 & 18,600 & 3.2 \\
1995 & 723,481 & 92.5 & 58,483 & 7.5 & 694,480 & 96.0 & 29,001 & 4.0 \\
\hline
\end{tabular}

Fuente: X y XI Censos Generales de Población y Vivienda, INEGI, 1984 y 1992; Conteo 1995, INEGI, 1996.

5 A mediados del siglo XIX se inició la construcción de "La Corriente", que fue el primer colector pluvial de la ciudad. Esta obra se construyó en torno al casco antiguo original, para conducir hacia el noreste la derrama pluvial que provenía de las corrientes naturales originadas en la sierra de San Miguelito, ubicada al sur de la ciudad. Este colector ha seguido operando a lo largo del siglo XX, con crecientes problemas debido a sus inadecuadas condiciones técnicas y al incremento de la derrama generada por descargas domiciliarias, que al sumarse a la descarga pluvial, generan constantes problemas e inundaciones.
Si bien entre 1980 y 1995 el porcentaje de población con este servicio aumentó de 75.6 a $92.5 \%$, la proporción de habitantes con desagüe al drenaje público disminuyó a lo largo del periodo, de 98.1 a $96.8 \%$. En contrapartida, la población con servicio de drenaje conectado a fosa séptica o con desagüe directo al suelo, se incrementó de manera constante, en una proporción superior a $400 \%$. De esa forma, e volumen de población que en 1995 carecía por completo del servicio de drenaje, sumado al que sólo contaba con fosa séptica o desagüe a suelo, alcanzaba una cifra cercana a los 90 mil habitantes, esto es, más de $11 \%$ de la población total del área metropolitana.

\section{Diferencias sociospaciales en la cobertura del servicio de agua potable}

Un aspecto colateral a los procesos señalados, pero no menos importante, es la existencia de marcadas disparidades en la cobertura y calidad de los servicios de agua potable y drenaje, que se expresa de manera diferencial conforme a los distintos sectores socioeconómicos de la población local, y a su ubicación al interior de la mancha urbana.

En el caso del servicio de agua potable, es evidente que el avance del desarrollo urbano a lo largo del tiempo ha significado, en términos generales, una mayor cobertura; sin embargo, algunos de los estratos sociales de la población metropolitana no han podido contar con este beneficio de la misma forma que otros grupos, generándose serias desigualdades en los grados de consumo, y en la accesibilidad y eficiencia del servicio.

Tal es la situación que afecta a innumerables colonias y fraccionamientos localizados al norte yoriente delárea metropolitana, donde el acceso a los servicios públicos llega a ser apremiante. De manera similar, pueden detectarse diversos sectores urbanos con serios padecimientos en esta materia. Así, por ejemplo, en torno al casco antiguo central y hacia el sur del primer contorno del centro histórico, se localiza un elevado número de vecindades, la mayoría de ellas habitadas y con múltiples deficiencias respecto a los recursos e infraestructura con que cuentan, especialmente en lo que concierne a la dotación de agua potable y drenaje.

Si se analiza a simple vista, la información del cuadro 4 puede resultar engañosa, ya que a principios de los años noventa, prácticamente la totalidad de la población residente en vecindades contaba con el servicio de agua potable. No obstante, una revisión más precisa nos indica que el déficit de este servicio dentro de las viviendas era $-\mathrm{y}$ sigue siendo-considerable; se aprecia así que, en 


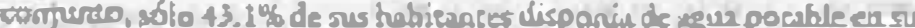

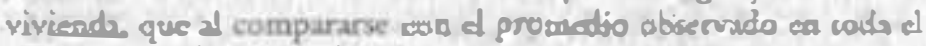

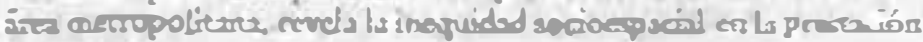
did sericio.

Curadro $\$$

ax de Sar Luis Pusosi. Cobarturs y disporibalidad de bred

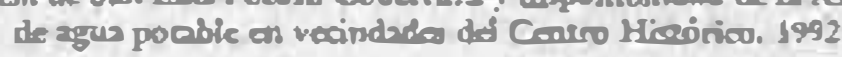

\begin{tabular}{|c|c|c|c|c|c|c|c|c|}
\hline Area/barrio & $\begin{array}{l}\text { Mosu ceo } \\
\text { servicio }\end{array}$ & s & $\begin{array}{l}\text { Mabe is } \\
\text { urioio }\end{array}$ & $=$ & $\begin{array}{l}\text { Donores } \\
\text { vivirady }\end{array}$ & $\%$ & $\begin{array}{l}\text { Fucral } \\
\text { viniends }\end{array}$ & s \\
\hline Tetal & (1)여 & $20=$ & 00 & as & +335 & 43.1 & 5.72t & ss. \\
\hline Zons Centes & $1.17:$ & \pm 208 & 0.0 & 0,0 & 214 & 221 & 938 & ret \\
\hline Ses Migartion & $198 \%$ & 100.6 & 0.0 & 00 & 665 & 32 & 1.121 & $\therefore$ \\
\hline Sxn Sebartian & 294 & 1050 & av & 00 & 100 & 30.1 & t,wi & ㄴ) 3 \\
\hline Merion & B5 & 102.0 & 00 & 0.0 & 52 & $38+4$ & 264 & 326 \\
\hline Thasals & 1.944 & 1028 & 0.0 & 0,0 & $1,4 \%$ & $i+, 9$ & 488 & 25,1 \\
\hline 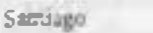 & $1.445^{\circ}$ & 500,0 & 00 & 0.0 & $5 \%$ & 378 & 911 & 62.1 \\
\hline
\end{tabular}

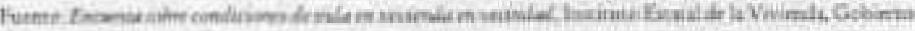

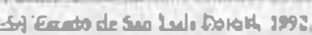

flys supuesto, ell slgunos casos, wamo son las vecindades ubicadrs én

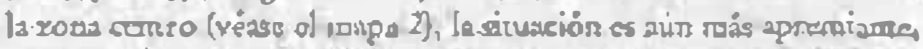

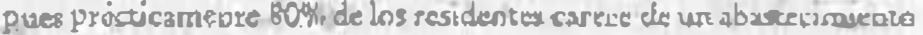
directo R su viviendo.

En oumnetuensia, us posible afirmur que, al igull que diverzss

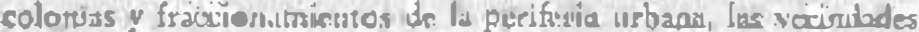
comtisponden a secto nes urbarsos que bran quedado fucea del procese

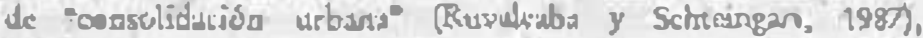

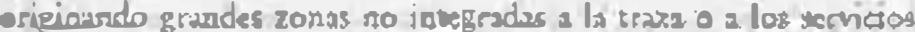

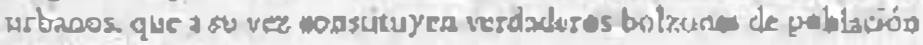
marzinoda

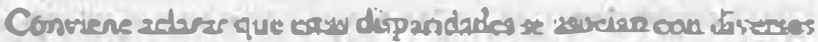

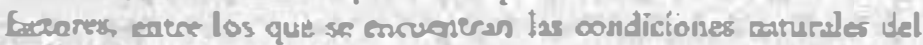

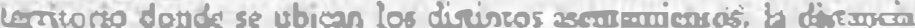
tortl y protradio que deben recorrer los usuncios paral zeoder al

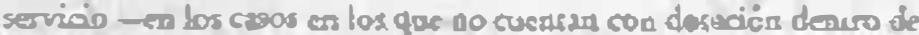

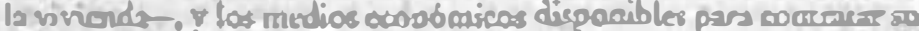

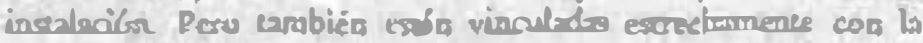

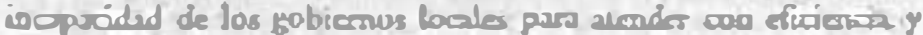

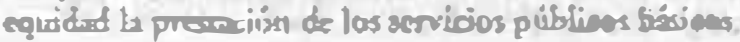

Mapa 2

Voxodarics del Como Historico. Sar I.uis Potosi.

Perioncuros "A " Barios tráfiogás

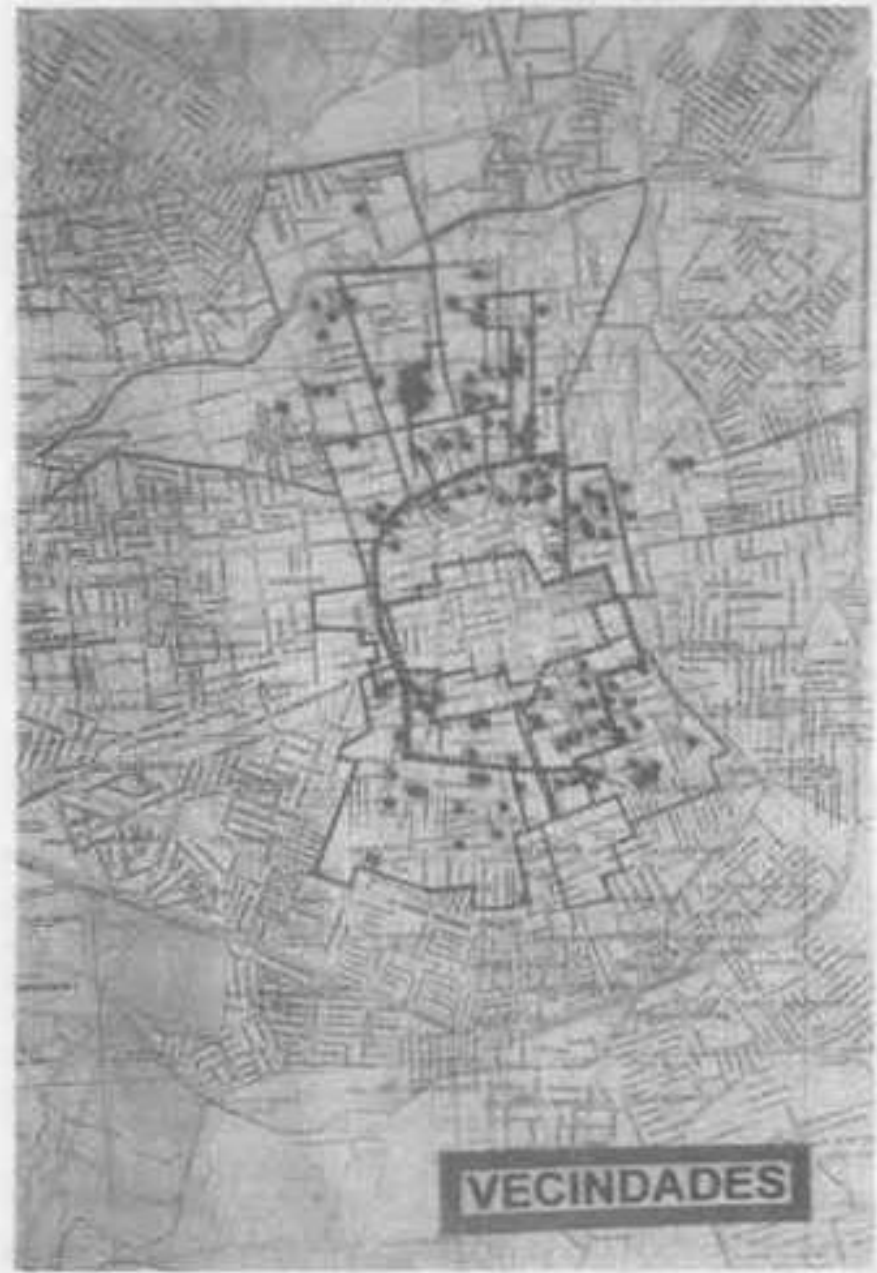


La organización y prestación de los servicios de agua potable y drenaje

Los servicios urbanos de agua potable y alcantarillado en la ZM de San Luis Potosí funcionan bajo la administración de la empresa Organismo Intermunicipal Metropolitano de Agua Potable, Alcantarillado, Saneamiento y Servicios Conexos (INTERAPAS), hasta hace poco denominada Sistema de Agua Potable y Saneamiento (SIAPAS). Esta empresa administra los sistemas de captación conducción, tratamiento, almacenamiento y distribución de agua potable, y los correspondientes a la red general de saneamiento. La operación descentralizada de este servicio tiene ya algún tiempo (SIAPAS fue creado en 1991, bajo la administración panista de Guillermo Pizzuto Zamanillo, e INTERAPAS comenzó a operar desde 1996, durante la gestión de Luis García Julián), por lo que no forma parte de los recientes intentos descentralizadores, ni es secuela del llamado "nuevo federalismo".

Sin embargo, la experiencia observada por este modelo de gestión de los servicios de agua potable y drenaje en estos siete años, puede resultar aleccionadora para las nuevas atribuciones que en la actualidad se intenta otorgar a los gobiernos locales, tanto de esta zona metropolitana, como de otras localidades urbanas del estado de San Luis Potosí. Con esta finalidad, a continuación se analizan algunos de los rasgos centrales de este modo de gestión.

\section{El modelo de gestión}

En primer lugar, se pudo verificar que la gestión del agua y el alcantarillado por parte de INTERAPAS, se concentra básicamente en el área técnica. Quizá por ello el organismo se ha visto sumamente limitado cuando ha debido encarar los efectos de la metropolización -tales como el violento crecimiento poblacional y la consecuente demanda de servicios-, o de la crisis económica prevaleciente -como el proceso inflacionario y la crisis financiera de los municipios de San Luis Potosí y Soledad de Graciano Sánchez.

Otro aspecto relevante derivado de este análisis consiste en la inexistencia de un control global sobre el proceso de gestión, en lo que se refiere a la producción y abastecimiento de agua, que se encuentra más bien limitado a la circunscripción de la empresa y, por tanto, no abarca toda el área metropolitana, ni otros sistemas de dotación existentes como: aprovisionamiento por medios privados - pipas o camiones cisterna, acarreo en botes-, la compra del líquido entre vecinos, acaparamiento, conexiones ilegales, entre otros. Una situación similar se observa sobre el proceso de gestión del sistema público de alcantarillado y drenaje.

En cuanto a las relaciones sociales y políticas que median en el proceso de gestión del sistema de agua potable y saneamiento, resulta interesante observar la presencia predominante de la burocracia del organismo, en sus facetas ingenieriles, administrativas y económico-financieras, en cuyas manos se concentran, casi de manera exclusiva, las principales decisiones que afectan la operación del servicio, por lo general bajo una racionalidad de carácter eminentemente técnica.

Sin embargo, no se puede omitir la intervención de otras entidades, que eventualmente participan en el nivel de la racionalidad política que requieren aspectos como la elevación de tarifas, o los planes técnicos o estratégicos que exige la construcción y licitación de plantas de tratamiento de aguas residuales. Mediante la participación de estos organismos se establecen negociaciones directas entre el gobierno estatal - por medio del Subcomité de Agua Potable y Saneamiento, del Comité para la Planeación del Desarrollo del Estado (COPLADE)-, dependencias federales como la Comisión Nacional del Agua (CNA), los gobiernos municipales, y quienes tienen en sus manos el control de cada uno de los sectores o fases de la gestión de los servicios.

Cabe aclarar que estas consultas o reuniones intersectoriales e interinstitucionales, por lo general son un mecanismo de la empresa para legitimar sus decisiones y evitar posibles bloqueos a sus argumentaciones técnicas. De ahí que prácticamente en todas las fases del proceso (planeación, inversión, ejecución, operación, seguimiento o evaluación del servicio), la racionalidad predominante también sea de corte técnico-empresarial, aunque ocasionalmente admite el juego de intereses políticos y económicos diversos, especialmente en lo que respecta a la fase de gestión política y social de los servicios.

Por supuesto, la participación de los usuarios aparece totalmente subordinada en este esquema, limitándose a ser actores pasivos y receptores de las disposiciones unilaterales de la empresa, sin dar lugar a reinvidicaciones legítimas; en ciertos casos, la presencia de la población en este escenario se reduce a la presentación de peticiones de ampliación del servicio, reclamos acerca de su eficiencia, o a su participación complementaria en alguna obra de interés colectivo mediante los Consejos de Desarrollo Municipal o Juntas de Mejoras. 
En suma, la identificación del modelo de gestión de ambos servicios públicos, es decir, de las distintas formas de organización de los recursos y de relación de los actores sociales y políticos involucrados en su prestación, permite caracterizarlo como un modo de gestión tecnocrático, en su vertiente tecno-burocrática (Velázquez, 1994) que, a diferencia del modo técnico-empresarial, caracterizado por un enfoque que busca trasladar al sector público los principios de la administración de la empresa privada y de la gerencia pública, introduce un matiz político en ciertos momentos de la gestión en el sentido de que los objetivos de productividad económica van acompañados por un auspicio del gasto público y por políticas sociales que se acogen a una lógica de las necesidades, y no de la productividad exclusivamente.

En el caso concreto de la operación de los servicios de agua potable y drenaje en la ZM de San Luis Potosí, los efectos de esta derivación del modelo tecnocrático tienden a reflejarse en lainjerencia de formas clientelares de relación entre la municipalidad y la población, con la participación de funcionarios municipales -con pretensiones electorales- o de intermediarios políticos, entre quienes destacan algunos líderes comunitarios.

En este contexto, es importante subrayar que la introducción de la variable política en la gestión, ha tenido diversos efectos sobre la prestación de los servicios públicos. Uno de ellos es la incorporación de nuevos usuarios al sistema, como producto del funcionamiento de redes clientelares o de una política asistencialista, más que de una concepción del carácter público del servicio.

A ello se agrega que, en términos operacionales, el modelo de gestión de los servicios de agua potable y saneamiento tiende a ser fuertemente rutinario e inmediatista, cumpliendo las tareas mínimas necesarias para su prestación. No existe, por tanto, un interés por innovar los sistemas de producción del servicio ni las formas de participación social. Evidentemente, esta situación dificulta las tareas de planificación, e inhibe la elaboración de estudios técnicos formales o planes maestros que permitan a las autoridades locales y al organismo operador, por un lado, prever nuevos desarrollos del servicio y mejorar su calidad, y por el otro, promover la modernización de los distintos procesos - administrativo, operacional, financiero y crediticio- que deben acompañar la adecuada prestación de estos servicios.

\section{Dependencia financiera, tamaño de la ciudad e inceppacidad técnica de los} municipios metropolitanos

En el marco descrito hasta aquí, encontramos evidencias de la problemática en la que se desenvuelve la prestación de los servicios públicos de agua potable y drenaje en la ZM de San Luis Potosí. Se han acotado ciertas dimensiones del problema, y explorado algunas posibles causas, que en parte explican los déficit registrados en ambos servicios durante los últimos años.

No obstante, una variable fundamental para analizar las limitaciones de losgobiernoslocales y del propio organismo operador para subsanar estas carencias, se relaciona con sus posibilidades reales de inversión. Al respecto, puede afirmarse que la operación descentralizada de estos servicios no ha significado el saneamiento financiero de la empresa administradora, la cual por cierto, ha tenido en distintos momentos serias crisis de endeudamiento y de transparencia en el manejo de recursos, solucionadas mediante subsidios del gobierno estatal y alzas en las tarifas.

De manera similar, la tendencia observada en los últimos años revela graves desequilibrios en las finanzas de los dos municipios metropolitanos, que permiten mantener el personal y el funcionamiento delos servicios en cuestión, pero que impiden realizar nuevos proyectos de inversión. Como se observa en el cuadro 5, entre 1989 y 1994 se apecia un crecimiento casi constante en la proporción de recursos invertidos en gasto corriente (gasto administrativo y servicios personales), que pasó de 40.4 a $47.3 \%$, mientras que el gasto de inversión (gasto en obra pública e infraestructura) se redujo durante el mismo lapso de 55.5 a 52.4 por ciento.

Es importante destacar que el valor absoluto más bajo alcanzado por el gasto en infraestructura y servicios públicos coincide con el inicio de la administración panista de Mario Leal Campos (1992). Posteriormente este nivel se recuperó ligeramente, a partir del apoyo que recibió el municipio de San Luis, mediante fondos del Programa Nacional deSolidaridad (PRONASOL) y del gobierno estatal.

Este hecho por sí mismo demuestra la limitada capacidad económica y financiera del gobierno de esta área metropolitana, que en realidad se encuentra determinada por los condicionamientos político-administrativos y de tramitación de recursos procedentes del gobierno central (federal y estatal), limitando sus posibilidades para desempeñar eficientemente las funciones que le corresponden, y enfrentar con éxito la creciente demanda en la dotación de servicios 
Cuadro 5

ZM de San Luis Potosí. Egresos de los municipios metropolitanos, gasto corriente y gasto de inversión, 1989-1994 (miles de pesos a precios constantes de 1994)

\begin{tabular}{||cccccccccc|}
\hline Año & $\begin{array}{c}\text { Egreso } \\
\text { total }\end{array}$ & $\%$ & $\begin{array}{c}\text { Gasto } \\
\text { corriente* }\end{array}$ & $\%$ & $\begin{array}{c}\text { Gasto } \\
\text { inversión } * *\end{array}$ & $\%$ & Otros & $\%$ \\
\hline 1989 & 67,546 & 100.00 & 27,272 & 40.38 & 37,488 & 55.50 & 2,786 & 4.12 \\
1990 & 79,795 & 100.00 & 33,328 & 41.77 & 44,662 & 55.97 & 1,805 & 2.26 \\
1991 & 94,253 & 100.00 & 39,703 & 42.12 & 54,444 & 57.76 & 0,106 & 0.12 \\
1992 & 100,867 & 100.00 & 50,589 & 50.15 & 46,429 & 46.03 & 3,849 & 3.82 \\
1993 & 106,134 & 100.00 & 50,908 & 47.97 & 46,716 & 44.02 & 8,510 & 8.01 \\
1994 & 128,139 & 100.00 & 60,577 & 47.27 & 67,095 & 52.36 & 0,467 & 0.37 \\
\hline
\end{tabular}

(*) Comprende gastos administrativos, servicios personales, honorarios, etcétera. (**) Incluye gasto en obra pública, infraestructura y servicios.

Fuente: Sistema de Información Municipal en Base de Datos (SMBAD), México, INEGI, 1996.

Cuadro 6

ZM de San Luis Potosí. Egresos de los municipios metropolitanos, gasto per cápita, 1989-1994

(pesos constantes de 1994)

\begin{tabular}{||ccc|}
\hline Año & Costo inversión & Inversión per cápita \\
\hline 1989 & 37,488 & 58.90 \\
1990 & 44,662 & 67.80 \\
1991 & 54,444 & 79.86 \\
1992 & 46,429 & 65.80 \\
1993 & 46,716 & 64.00 \\
1994 & 67,095 & 88.80 \\
\hline
\end{tabular}

Fuente: Sistema de Información Municipal en Base de Datos (SMBAD), INEGI, México, 1996. básicos, no obstante que los recursos casi se duplicaron durante el periodo 1989-1994.

Para justificar las deficiencias señaladas, los gobiernos de municipios urbanos tradicionalmente han esgrimido el argumento del impacto desfavorable del crecimiento demográfico en las finanzas municipales, y por ende, en el monto de recursos invertidos en materia de infraestructura y servicios públicos. $\mathrm{Al}$ respecto, diversos autores (Ladd, 1990 y 1992; Synder y Stegman, 1987; entre otros) han encontrado que, efectivamente, el rápido crecimiento de la población de una ciudad, y el consecuente incremento en la intensidad o densidad de uso del suelo, pueden traer consigo efectos fiscales indeseables que incrementan los costos de provisión de los servicios públicos, demeritando la calidad y cobertura de los mismos.

En el caso que nos ocupa, la información disponible en el cuadro 6 revela que entre 1989 y 1994 la inversión absoluta y per cápita en materia de infraestructura y servicios se incrementó de 1989 a 1991, cayendo entre este último año y 1993, y recuperándose en 1994. Así, al inicio del periodo estudiado, los gobiernos de la zona metropolitana invertían de manera conjunta 58.90 pesos por habitante anualmente, mientras que en 1994 esta cifra ascendió a 88.80 pesos.

Lo anterior significa que la inversión pública en esta materia se ajustó rápidamente al crecimiento de la población. Por tanto, el argumento de justificar la baja calidad y las deficiencias en la cobertura de los servicios de agua potable y drenaje debido al crecimiento de la población de la zona, difícilmente puede ser válido, o por lo menos podría considerarse ambiguo. Lo que síllama la aténción es la caída en el gasto total durante los dos primeros años de la administración del panista Mario Leal Campos; caída originada fundamentalmente por una disminución de las participaciones federales hacia el municipio de San Luis Potosí.

\section{Conclusiones y recomendaciones}

Los resultados expuestos permiten confirmar, aunque sea de manera tentativa, algunos de los supuestos iniciales acerca de la (in)capacidad de los gobiernos locales en la administración y gestión de los servicios públicos, entendidas como la combinación de actuaciones y trámites que son necesarios para asegurar el desarrollo ordenado y eficaz de esta zona metropolitana, y para garantizar el mejoramiento de las condiciones de vida y de trabajo de sus habitantes. 
En primer término, se encontró que la alternancia en el control de las administraciones municipales por parte del PRI, del PAN, o de otros partidos o coaliciones, no ha significado, en nuestro caso de estudio, necesariamente una mejor gestión de los servicios públicos, pero sí cambios en sus finanzas locales. Los gobiernos municipales entrantes han tenido que absorber un serio desgaste, en términos de legitimidad y gobernabilidad, derivado en gran medida de las dificultades que han encontrado para enfrentar el problema de los servicios de agua potable y drenaje; lo cual, cabe decir, no necesariamente se ha reflejado en la arena político-electoral. ${ }^{6}$

Lo mismo sucede con el traspaso de atribuciones, recursos y responsabilidades hacia los gobiernos locales, que independientemente de su filiación partidista, han puesto de relieve la enorme dependencia de éstos respecto a los gobiernos federal o estatal; aspecto que constituye uno de los problemas torales del gobierno metropolitano de San Luis Potosí.

En este sentido, es conveniente subrayar que la carencia de ingresos que padecen los municipios estudiados no define por sí misma su dependencia financiera. Sus rezagos en materia de autonomía, descentralización, y eficiencia administrativa o técnica, tienen otros componentes más profundos, tales como su relación clientelar y subordinada a los otros sectores del gobierno, ${ }^{7}$ las características e inercias de su estructura interna, y la ausencia de mecanismos de participación ciudadana en los procesos de planeación, administración, gestión y evaluación de los servicios públicos.

En relación con este punto, la información revisada revela serias deficiencias en el aparato planificador metropolitano local, que

${ }^{6}$ En las recientes elecciones municipales de julio, el ganador fue el candidato del PAN, quien venció por un cerrado margen al candidato del partido oficial. En contrapartida, las elecciones para gobernador del estado dieron el triunfo al candidato del PRI. Esto revela, por un lado, el carácter muchas veces veleidoso de la decisión ciudadana al votar por uno u otro partido; decisión que a simple vista puede contradecir las reflexiones más sesudas, pero que en este caso se relacionan con dos factores ampliamente comentados por los politólo ques nacionales: el "voto de castigo" al PRI y la preferencia por el perfil del candidato sobre el perfil 0 ideario partidista.

${ }^{7}$ Un claro ejemplo de esta situación lo constituyen las obras del Colector Vial Reforma, cuya ejecución fue postergada por el gobierno municipal panista de Mario Leal Campos, que habia incion gobernador Horacio Sanchez Unzueta dio su anuencia, previa a los procesos electorales de 1997, para la autorización y liberación de recursos federales y estatales que permitieron iniciarla casi al concluir la gestión priista de Luis García Julian. Obra que, por cierto, no verán concluida en su totalidad duante su gestión ni el gobierno estatal ni el municipal en turno, dejándola como una "herencia" más a las administraciones entrantes. se expresan en la falta de previsión y de perspectiva a largo plazo en la administración, planeación y gestión de los servicios de agua potable y saneamiento. ${ }^{8}$

En consecuencia, al igual que en otros aspectos, en materia de servicios públicos no existe una propuesta o estrategia de desarrollo, mucho menos de carácter metropolitano, sino un enfoque reactivo que se limita a la solución de las carencias o déficit en las áreas de reciente urbanización, $y$ al mantenimiento, construcción y funcionamiento de los servicios en general. Sin embargo, deja fuera aspectos relacionados con una estrategia económica urbana, el problema del desempleo o subempleo, la refuncionalización de los usos del suelo y el control del mercado especulativo del suelo, el desarrollo urbano sustentable, o simplemente la incorporación de formas participativas en la gestión de los servicios públicos.

Por el contrario, especialmente en el caso de las administraciones panistas, esta situación ha agudizado una gran apertura a esquemas de carácter eminentemente técnico, de origen ideológico neoliberal -reaganiano y thatcheriano-, plasmados en la instrumentación de modelos de administración de los servicios públicos municipales que permiten adoptar modalidades de prestación basadas en la privatización y descentralización de los mismos (Herrera, 1995; Soria, 1995), pero que no consideran los criterios de equidad y de participación comunitaria en la gestión de estos servicios.

Como resultado, se ha impuesto un modelo de gestión tecnoburocrático, cuyos principales adeptos han sido los gobiernos municipales panistas, pero también grupos privados, locales o foráneos, que han percibido en estos esquemas de privatización, la existencia de un importante componente de rentabilidad.

Este aspecto cobra una gran relevancia para la imagen que pretenden ofrecer dichas administraciones a la ciudadanía, ya que en términos ideológicos la privatización de los servicios de agua potable y drenaje se plantea como una supuesta innovación, pero además, en la práctica, independientemente de su nulo reflejo en la eficiencia o eficacia de la prestación de esos servicios, les permiten descargar gran

${ }^{8}$ A pesar de la incorporación de la función planificadora del municipio, desde el punto de vista jurídico-formal, con la creación del Instituto Municipal de Planesción Urbana en 1996, que en el papel constituyó una interesante innovación, en la práctica su operación presenta aún serias limitaciones. Si bien el decreto de creación de este organismo define como objetivos centrales el ejercicio eficaz de planificación, la gestión de proyectos y la obtención de recursos, el desempeño de estas funciones prácticamente ha estado ausente desde su de recursos, el desempeño de estas funciones prácticamente ha estado ausente desde su
instalación, limitándose a elaborar diagnósticos generales e identificar posibles proyectos de inversión, carentes de una política de mediano o largo plazos. 
parte de sus responsabilidades, tanto en el nivel económico, como en el administrativo y político.

En este punto, y a manera de corolario, podrían plantearse algunos aspectos fundamentales sobre la gestión municipal de esta zona metropolitana, tales como: ¿Qué orientación del desarrollo, en general, y de sus diversas vertientes, en particular, deben seguir los municipios metropolitanos? ¿Bajo qué criterios pueden conciliarse en la práctica los reclamos populares con los requerimientos de largo plazo para el desarrollo de la infraestructura y los servicios? ¿Cómo puede sumarse de manera efectiva y democrática la iniciativa ciudadana a la gestión de los servicios? ¿Cómo puede establecerse y mantenerse la gobernabilidad municipal sin caer en procesos clientelares? ¿Cuál es la toma de decisiones adecuada en la asignación de recursos?

Estos temas implican a su vez una necesaria preocupación por el desarrollo municipal y metropolitano, y no sólo por la administración de la escasez. Evidentemente, el problema de la suficiencia financiera y presupuestal es de la mayor importancia. No obstante, paradójicamente, mientras menores sean los recursos disponibles, más adecuado debe ser su manejo y distribución.

Por otro lado, la emergencia de la sociedad civil, en su creciente búsqueda de espacios de expresión, representa también una oportunidad para movilizar una gran cantidad y diversidad de recursos. De aquí se deduce que la convocatoria a la ciudadanía y sus organizaciones para contribuir a la promoción del desarrollo municipal, requiere también de un planteamiento claro y, sobre todo, con visión de largo plazo.

En consecuencia, la eficiencia y eficacia del municipio radica cada vez más en la capacidad de planear adecuadamente su gestión. Es decir, contar con un proceso adecuado de toma de decisiones, que incorpore las iniciativas de los grupos sociales y que permita conciliar el desarrollo de largo plazo con la atención de demandas inmediatas, en un clima de gobernabilidad y transparencia de la gestión local. Ello implica una necesaria redefinición del ámbito de la planificación del desarrollo municipal, a partir de un modelo en el que se mida la actuación institucional de los órganos planificadores municipales, y de los instrumentos destinados a la prestación y gestión de los servicios públicos básicos.

En este sentido, se hace evidente la necesidad de dejar atrás una visión en la que prevalece la noción del planificador urbano y el administrador público, como simples "ejecutores de los rígidos planes gubernamentales [...] para dar lugar al gestor o gerente público, con capacidad emprendedora y generadora de consensos, en marcos interinstitucionales móviles y conflictivos, en un proceso planificador por aproximaciones sucesivas, en el que el valor esperado es el proceso mismo y no el producto final llamado plan" (Cabrero, 1995:2). ${ }^{9}$

Finalmente, conviene aclarar que la evaluación que en este documento se presenta posee un carácter eminentemente aproximativo, basado primordialmente en aspectos e información relativos a un caso de estudio como es la ZM de San Luis Potosí. Por tanto, debe interpretarse con reservas, precisamente porque los resultados de este trabajo pueden contrastar con la gran diversidad de circunstancias y condiciones que en el ámbito local hacen de cada municipio en México un caso especial.

\section{Bibliografía}

Aguilar Robledo, Miguel (1997), "El debate modernidad/postmodernidad y la renovación del discurso de la planeación", Revista Interamericana de Planificación, SIAP.

Cabrero Mendoza, Enrique (1994), La gestión de servicios urbanos: un reto a la innovación en los gobiernos locales, documento de trabajo, México, CIDE.

(1995), La nueva gestión municipal en México. Análisis de experiencias innovadoras en gobiernos locales, México, CIDE.

Comisión Nacional del Agua (CNA) (1994), Desarrollo Hidráulico del Valle de San Luis Potosí (Datos Básicos), San Luis Potosí, CNA/Gerencia Estatal San Luis Potosí, marzo.

Herrera, Alejandro (1995), "La privatización de servicios públicos como una política pública alternativa", Federalismo $y$

${ }^{9} \mathrm{Al}$ respecto, es importante destacar que la planeación moderna, como algunas otras disciplinas, está involucrada en el actual debate modernidad/posmodernidad, a partir del cual, como bien señala Miguel Aguilar, "la disciplina está enfrentando una profunda crisis, expresada en un proceso de ajuste que afecta su enfoque, función e identidad disciplinaria. Así, la planeación está experimentando una transicion desde una perspectiva comprehensiva hacia una más estratégica; desde un enfoque central o regional hacia uno local; desde una visión 'desde arriba' hacia una 'desde abajo'; y por último, pero no menos importante, de aportar la racionalidad para la intervención del Estado en un territorio específico, hacia una perspectiva basada en la construcción de consensos" (Aguilar, 1997:1). 
Desarrollo, México, BANOBRAS, año 9, núm. 51, septiembreoctubre, pp. 31-34.

INEgI (1986), X Censo General de Población y Vivienda, México, INEGI.

(1990), XI Censo General de Población y Vivienda, México, INEGI.

(1995), Conteo 1995. Resultados Finales, Aguascalientes, INEGI.

- (1996), Soledad de Graciano Sánchez, Estadode San Luis Potosí. Cuaderno Estadístico Municipal, Aguascalientes, INEGI.

(1997), San Luis Potosí. Estado de San Luis Potosí. Cuaderno Estadístico Municipal, Aguascalientes, INEGI.

Ladd, Helen (1990), "Effects of population growth on local spending and taxes", en Proceedings of the 1990 Conference of the Association for Public Policy Analysis and Management, San Francisco, Calif.

(1992), "Population Growth, Density and the Costs of Providing Public Services”, Urban Studies, vol. 29, núm. 2, pp. 273-295.

Niedzielski, Henryk (1990), "Perspectivas del suministro de agua a la ciudad de San Luis Potosí", Ciencia, vol. 41, núm. 2, pp. 153162.

Ruvalcaba, Rosa Ma. y Martha Schteingart (1987), "Estructura urbana y diferenciación socioespacial en la zona metropolitana de la ciudad de México (1970-1980)", en Atlas de la Ciudad de México, México, DDF-El Colegio de México.

Soria Romo, Rigoberto (1995), "Hacia un modelo alternativo en el financiamiento y prestación de los servicios municipales", Federalismo y Desarrollo, México, BANOBRAS, año 9, núm. 51, septiembre-octubre, pp. 48-73.

Synder, T. y M. Stegman (1987), Paying for Growth, Washington, D. C., Urban Land Institute.
Velázquez, Fabio C. (1994), "La gestión local de servicios públicos. Consideraciones finales", en A. Rodríguez y F. Velázquez, Municipio y servicios públicos. Gobiernos locales en ciudades intermediasde América Latina, Medellín, Colombia,Ediciones Sur. 